\title{
Needle Shape Estimation in Soft Tissue Based on Partial Ultrasound Image Observation
}

\author{
Jay Carriere ${ }^{1}$, Carlos Rossa ${ }^{1}$, Nawaid Usmani ${ }^{2}$, Ronald Sloboda ${ }^{2}$, Mahdi Tavakoli ${ }^{1}$
}

\begin{abstract}
We propose a method to estimate the entire shape of a long flexible needle, suitable for a needle insertion assistant robot. This method bases its prediction on only a small segment of a needle, imaged via ultrasound, after insertion. An algorithm is developed that can segment a needle observed partially in ultrasound images and fully in camera images, returning a polynomial representation of the needle shape after RANSAC processing. The polynomial corresponding to the partial needle observation in ultrasound images is used as the input to a needle-tissue interaction model that predicts the entire needle shape. The needle shape predicted by the model is compared to the segmented needle shape based on camera images to validate the proposed approach. The results show that the entire needle shape can be accurately predicted in tissues of varying stiffness based on observation of parts of the needle in an ultrasound image.
\end{abstract}

\section{INTRODUCTION}

Prostate brachytherapy is a procedure whereby long flexible needles containing radioactive seeds are inserted under ultrasound guidance into the prostate to treat cancerous tissue. The success of this surgery depends on the accuracy with which the seeds are placed within the prostate with respect to pre-planned target locations [1]. During insertion, however, each needle will bend away from its target location, requiring continuous observation and correction of the needle path.

A typical brachytherapy setup, diagrammed in Fig. 1, includes an ultrasound probe registered to a guide template that consists of a grid of holes. Each brachytherapy needle is inserted through a grid hole that corresponds to a pre-planned target location in the prostate. During needle insertion, an ultrasound image at the specified target depth is acquired to check the needle position. Only a small portion of the needle can be imaged at any time as the field of view of the ultrasound is very narrow. Thus, as the needle is sent off course from its target, the surgeon is unable to see or predict the needle's total deflection. Needle shape prediction has traditionally been done using mechanically based models that require tissue parameters as an input [2], [3], [4].

This work was supported by the Natural Sciences and Engineering Research Council (NSERC) of Canada under grant CHRP 446520, the Canadian Institutes of Health Research (CIHR) under grant CPG 127768, and by the Alberta Innovates - Health Solutions (AIHS) under grant CRIO 201201232.

${ }^{1}$ Jay Carriere, Carlos Rossa, and Mahdi Tavakoli (Corresponding Author) are with the Department of Electrical and Computer Engineering, University of Alberta, AB, Canada T6G 2V4. E-mail: jtcarrie@ualberta.ca; rossa@ualberta.ca; mahdi.tavakoli@ualberta.ca.

${ }^{2}$ Ronald Sloboda and Nawaid Usmani are with the Cross Cancer Institute and the Department of Oncology, University of Alberta, Edmonton, AB, Canada T6G 1Z2. E-mail: ron.sloboda@albertahealthservices.ca; nawaid.usmani@albertahealthservices.ca.

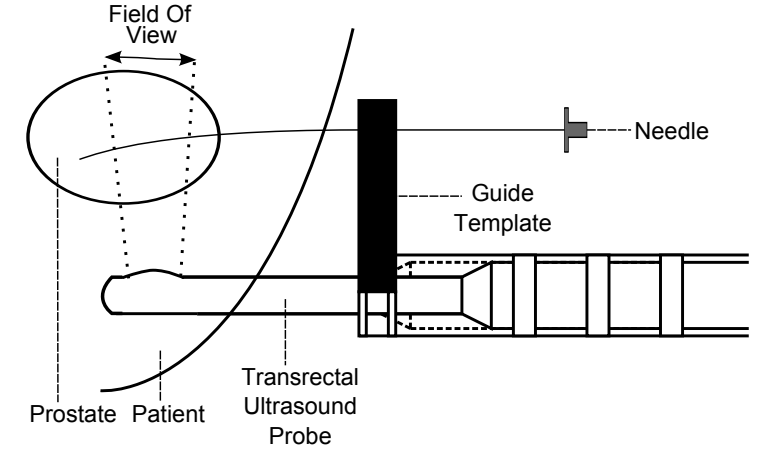

Fig. 1. Typical brachytherapy setup including ultrasound probe and perineal template

Additionally some models depend upon knowledge of other parameters, for instance the expended mechanical work to insert the needle [2], the needle and tissue interaction force profiles [2], [3], or complex finite element tissue deformation models [4]. Instead of using tissue characterization and complex mechanical interaction profiles, in this paper we have developed a predictive algorithm that only requires data gathered from partial needle observation in an ultrasound image and the basic mechanical characteristics of the needle.

The first step in predicting the needle shape in our method is to acquire ultrasound images of a portion of the brachytherapy needle inserted into tissue (Section II). Next, we introduce a novel technique for segmenting bent needle images by combining edge detection and thresholding techniques with RANdom SAmple Consensus (RANSAC) filtering [5]. The segmentation algorithm outputs a second order polynomial that fits to the portion of the needle seen in the ultrasound image. This needle-tissue interaction model is then used to predict the entire needle shape from the observed portion of the needle seen in the ultrasound image (Section III). The input polynomials are required to be converted by an affine transformation into the real-world frame so that they can be used by the needle-tissue interaction model and to be in a common reference frame for validation (Section IV). Finally, to validate this prediction we compare the shape output from the model with the shape of the needle as seen in the camera images (Section V), which represents the ground truth. The overall flowchart of the algorithm is given in Fig. 2 .

\section{CAMERA AND Ultrasound ImAGE PRocessing}

Given that our primary goal is to predict the total needle shape from the observation of a small portion of the needle, 


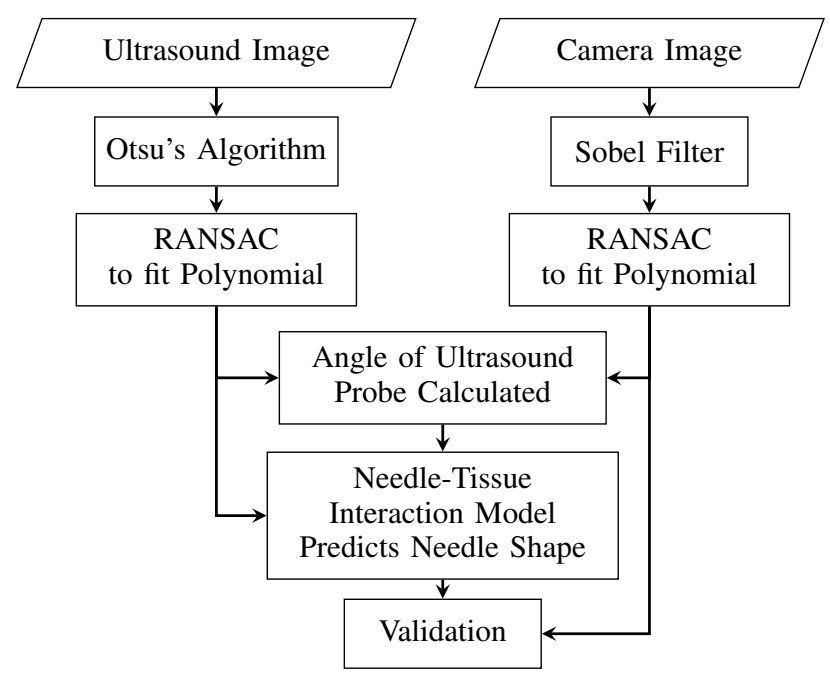

Fig. 2. Needle Prediction and Validation Algorithm

in this section we present a technique that is used to find a curved needle in an ultrasound image. This technique for segmenting a curved needle in an ultrasound is then extended to a camera image. The needle segmentation algorithm results in a second order polynomial equation that follows the curvature of the needle in its respective frame of reference, i.e. either the camera frame or the ultrasound image frame.

The experimental setup consists of a tissue phantom imaged from overhead by a camera and from the side by an ultrasound probe. The images show a needle that is inserted into the tissue that deflected during insertion. Fig. 3 shows a schematic representation of the experimental set up in order to define the different coordinate frames that arise from combining imaging modalities. Points in both the camera and ultrasound images are throughout the paper defined in their respective pixel domains. In the convention here, superscripts and subscripts on the left side of a symbol refer to the frame of reference for the coordinate system. The coordinate frame for the ultrasound image is denoted by $U$; a point ${ }^{U} P$ in the ultrasound image will have coordinates $\left({ }^{U} x,{ }^{U} y\right)$ defined with respect to the lower left hand side of the ultrasound image, as shown in Fig. 3. The coordinate frame for the camera image is denoted by $C$; a point ${ }^{C} P$ in the camera image will have coordinates $\left({ }^{C} x,{ }^{C} y\right)$, defined with respect to the upper left hand side of the camera image, as indicated in Fig. 3. A third frame is defined not in a pixel domain but in physical coordinates and referred to as the real-world frame, denoted by $R$. A point ${ }^{R} P$ in the real-world frame consists of $\left({ }^{R} x,{ }^{R} y\right)$ measured in meters with respect to an origin located where the needle exits the template (see Fig. 3).

\section{A. Ultrasound Image Processing}

Needle segmentation in 2D ultrasound images has previously been done via Gabor Filtering [6] and the Hough Transform for straight needles [7] or curved needles [8]. Additionally, needles have been segmented in 3D ultrasound images using projection methods [9], [10] or RANSAC [11].

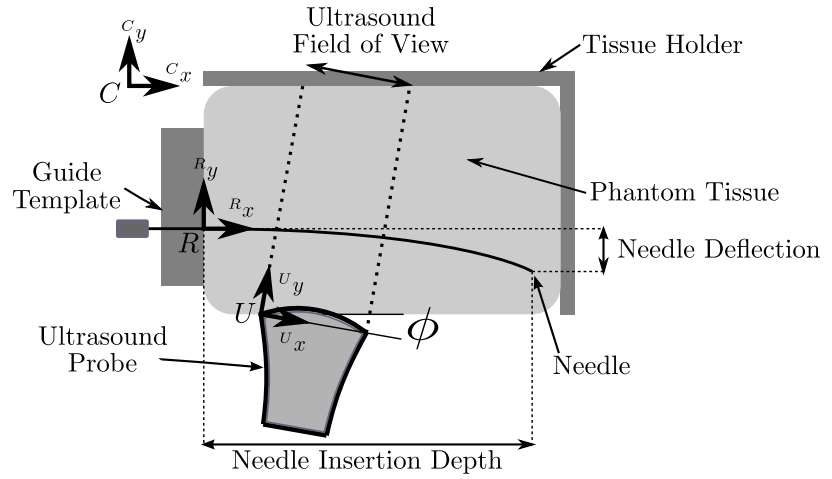

Fig. 3. Brachytherapy procedure simulation setup including ultrasound probe, phantom tissue, needle template, and needle. The setup is represented from the field of view of the camera.

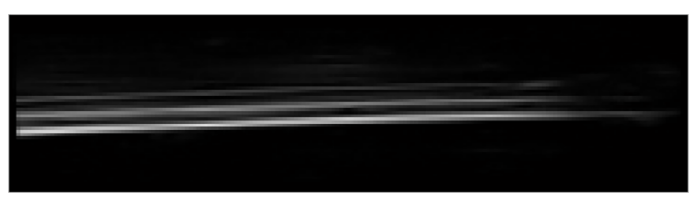

(a) Input Ultrasound Image

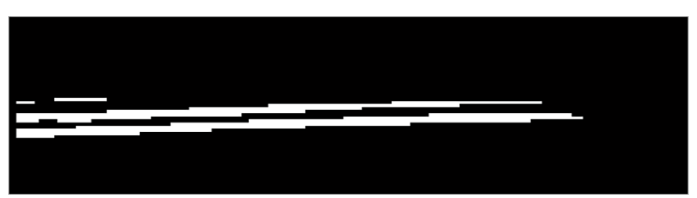

(b) Ultrasound Image After Image Processing

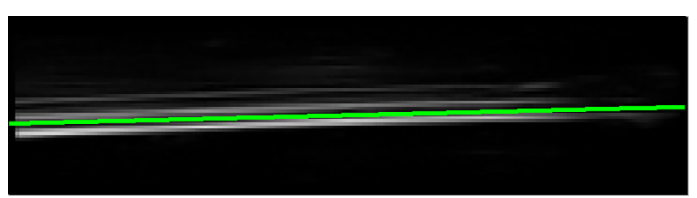

(c) Polynomial Curve Fit to Needle in Ultrasound Image

Fig. 4. Image Processing for Ultrasound

In this paper, we combine RANSAC filtering with Otsu's Algorithm [12].

Ultrasound images along the length of the needle are taken near the needle's entry point into tissue, as this represents the most challenging scenario for prediction. The probe used to capture the images is a linear transducer and as such shows a limited field of view, in this case a linear $40 \mathrm{~mm}$ wide image that does not widen with depth. These images are captured at arbitrary distances from 2 to $66 \mathrm{~mm}$ away from the needle's entry point into the tissue. When the needle is imaged under ultrasound while in phantom tissue, the needle is quite distinct from the background as shown in Fig. 4(a). The routine for segmenting the needle in an ultrasound image involves thresholding using Otsu's algorithm [12]. This converts the grayscale ultrasound image into a segmented binary (black and white) image, as shown in Fig. 4(b). The white values in the binary image are converted into a list of points ${ }^{U} P$, which is the input for the RANSAC algorithm. Image processing is implemented in Matlab using the functions from the image processing toolbox. 


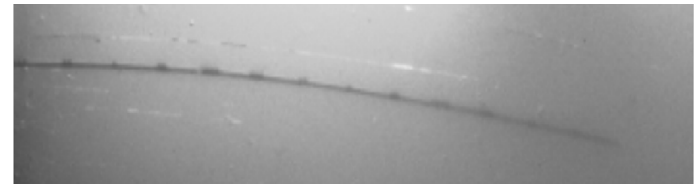

(a) Input Camera Image

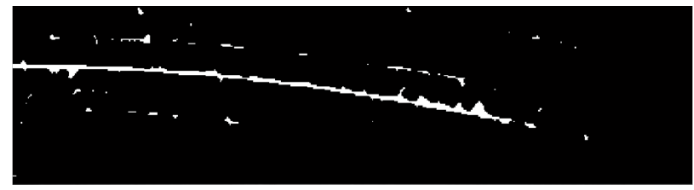

(b) Camera Image After Image Processing

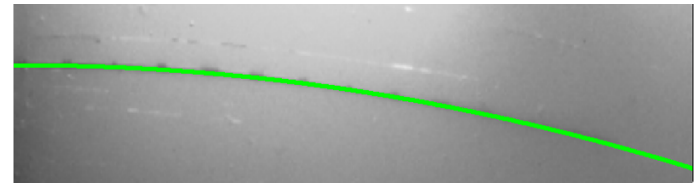

(c) Polynomial Curve Fit to Needle in Camera Image

Fig. 5. Image Processing for Camera Image

\section{B. Camera Image Processing}

Image processing is performed on the camera image which will be used later for model validation. In contrast to the ultrasound image, the needle in the camera image is not readily segmentable based on a grayscale threshold as the image suffers from variations in lighting intensity which can be seen in Fig. 5(a). Therefore, segmenting the needle from the camera image is performed using the Sobel edge detection filter, a $3 \times 3$ gradient filter that approximates the 2D derivative of the image with respect to image intensity [13]. From this, a threshold is applied to the filtered image (i.e., the $2 \mathrm{D}$ derivative) and only those edges exceeding the threshold are accepted, thus creating a binary image. The output of the camera image processing is shown in Fig. 5(b). After the Sobel filter is applied to the image, the white values are converted to a list of points ${ }^{C} P$ to be input to perform RANSAC filtering on the image.

\section{Processed Image Conversion to Polynomial via RANSAC}

The next step in the algorithm is to convert a list of segmented points from the binary images into polynomial curves. RANSAC or RANdom SAmple Consensus [5] is the algorithm that we will use to fit polynomials as it is robust to noise. The input to the RANSAC algorithm is a collection of points from either the camera ${ }^{C} P$ or ultrasound ${ }^{U} P$, in their respective frames, that represent possible points along the needle. We want to convert the points from ${ }^{C} P$ and ${ }^{U} P$ into second order polynomials

$$
\begin{gathered}
{ }^{C} y=\alpha_{2}\left({ }^{C} x\right)^{2}+\alpha_{1}\left({ }^{C} x\right)+\alpha_{0} \\
{ }^{U} y=\beta_{2}\left({ }^{U} x\right)^{2}+\beta_{1}\left({ }^{U} x\right)+\beta_{0}
\end{gathered}
$$

where the $\alpha$ and $\beta$ terms are the coefficients of the desired polynomials that best describe the needle.

The RANSAC algorithm takes 3 random points from the sets ${ }^{C} P$ or ${ }^{U} P$ and uses linear least squares to calculate the

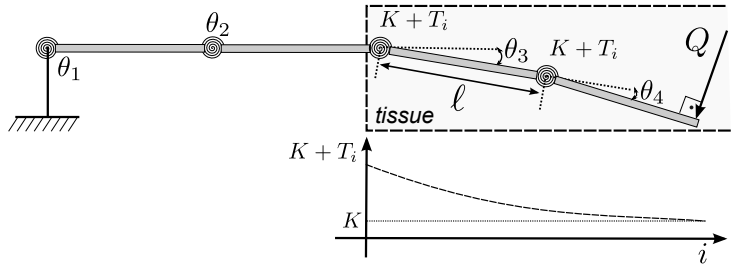

Fig. 6. Needle-tissue interaction lumped model. The length of the needle is represented by discrete weightless bars $\ell$

polynomial coefficients in (1) and (2) respectively. Outliers are defined as points that are further than a given distance from the curves (1) and (2). The ratio of inliers to outliers is then calculated and if this ratio is greater than a threshold the algorithm calculates the coefficients for polynomials (1) and (2) considering the entire set of inlier points. If the ratio is less than the threshold, then three different points from ${ }^{C} P$ or ${ }^{U} P$ are chosen and the algorithm restarts. For our implementation we use maximum distance values of 3 pixels for the camera image and 7 pixels for ultrasound image. For the inlier to outlier ratio we use $70 \%$ and $80 \%$ for the camera image and ultrasound image respectively. After the RANSAC routine has successfully been run on the two sets of points ${ }^{C} P$ and ${ }^{U} P$, the resulting polynomials follow the curve of the needle in either image. The results after RANSAC polynomial curve fitting are shown in Fig. 4(c) and Fig. 5(c).

\section{NeEdle-Tissue Interaction Model}

In order to predict the curvature of the needle based on observing only a portion of the needle we will use a physical model that incorporates characteristics of both the needle and the phantom tissue. This model is an extension of [14]. The model is a non-linear needle deflection estimator using only the portion of the needle imaged by ultrasound (converted into the real-world coordinate frame, see Section IV), needle insertion length, and the needle mechanical properties as its inputs. The total inserted length of the needle $L$ is discretized into $n$ non-deformable weightless bars of length $\ell$ such that $\ell=L / n$, as shown in Fig. 6 . Let us define $\theta_{i}$ as the relative angle between a bar segment of the needle $i-1$ and the subsequent bar segment $i$. Two neighboring bar segments $i-1$ and $i$ are considered to be connected by helical springs, all of which have the stiffness $K$, given by

$$
K=\frac{E I}{L} n
$$

where $E$ and $I$ are the needle Young's modulus of elasticity and its second moment of inertia. As the needle is inserted into the tissue, a force $Q$ is applied to the tip of the needle perpendicular to last segment, such that the needle bends. As the needle bends during insertion it increasingly compresses the tissue located under the needle, this in turn causes the tissue to apply a reaction force back onto the needle. The actions of these forces are modeled as helical springs of stiffness $T_{i}$, which is added to the original spring stiffness of $K$. Together, these two stiffnesses resist the deflection of 
the needle. This results in the helical springs inside the tissue having a stiffness of $K+T_{i}$. The stiffness $T_{i}$ is modeled as

$$
T_{i}=k_{0}[\ell(n-i+1)]^{k_{1}}
$$

where $k_{0}$ and $k_{1}$ are variables that depend on tissue parameters. Together $k_{0}, k_{1}$, and $Q$ represent the model parameters that will be found interactively using a pattern search algorithm implemented in Matlab.

To obtain the needle deflection we consider the torque $\tau_{i}$ generated on each joint $i$. The torque $\tau_{i}$ is a result of the effect of the needle tip force $Q$, and its influence at each joint is $\tau_{i}=$ $Q G_{i}$, where $1 \leq i \leq n$ and $G_{i}$ is a Jacobian transformation. The components $G_{i}$ of the Jacobian matrix relating the force $Q$ to the relative angles $\tau_{i}$ as $\tau_{i}=G_{i} F_{t}$ are

$$
G_{i}=\ell\left(1+\sum_{p=i}^{n} \cos \theta_{p}-\cos \theta_{i}\right) \cos \left(\sum_{p=i}^{n} \theta_{p}-\theta_{i}\right)
$$

Thus, the relative angular displacement for each of the segments can be calculated as $\theta_{i}=\tau_{i} / K_{i}$. Finally, we can calculate the Cartesian position of each helical spring in the transverse $\left(v_{i}\right)$ and axial $\left(u_{i}\right)$ directions (with respect to the unbent needle) as

$$
\begin{aligned}
& v_{i}=v_{i-1}+\ell \sin \left(\sum_{p=1}^{i} \theta_{p}\right) \\
& u_{i}=u_{i-1}+\ell \cos \left(\sum_{p=1}^{i} \theta_{p}\right)
\end{aligned}
$$

Solving for the needle's total deflection requires knowledge of the needle tip force $Q$ and the tissue reaction force as modeled by $k_{0}$ and $k_{1}$, from (4). Since these equations cannot be solved analytically, we need to use an iterative method to find a solution. In order to do that the polynomial coefficients of the needle from the ultrasound image are used to generate a series of points in the real-world frame, originally from ${ }^{C} P$, that represent a portion of the needle. The points in the real-world frame will be known as ${ }^{R} P_{U}$ having coordinates $\left({ }^{R} x_{U},{ }^{R} y_{U}\right)$ in the axial and transverse direction and are found by taking points from the polynomial (2) and transforming them through ${ }_{U}^{R} T$ such that a new polynomial is created that describes the deflection seen by the ultrasound probe in the real frame, given by

$$
{ }^{R} y_{U}=\gamma_{2}\left({ }^{R} x_{U}\right)^{2}+\gamma_{1}\left({ }^{R} x_{U}\right)+\gamma_{0}
$$

where $\gamma_{2}, \gamma_{1}, \gamma_{0}$ are fit to the transformed points by linear least squares.

The values of $Q, k_{0}$, and $k_{1}$ are found through minimizing the following cost function using a pattern search algorithm

$$
C\left(Q, k_{0}, k_{1}\right)=\sum_{i=j_{1}}^{j_{2}}\left|v_{i}\left(Q, k_{0}, k_{1}\right)-{ }^{R} y_{U i}\right|
$$

where ${ }^{R} y_{U i}$ are discrete sample points taken from (8), such that ${ }^{R} x_{U i}=u_{i}$ for every element $i$ that is in the portion of the needle simulation corresponding to the same portion of the needle imaged under ultrasound; thus $j_{1} \leq i \leq j_{2}$, with $j_{1}$ and $j_{2}$ being the boundaries on the left and right side of the needle portion respectively. $v_{i}\left(Q, k_{0}, k_{1}\right)$ is the simulated needle deflection at the corresponding axial position $u_{i}$ for a given set of $Q, k_{0}$, and $k_{1}$. The values of $Q, k_{0}$, and $k_{1}$ are iteratively changed to minimize the cost function and thus find the model parameters. With these parameters found, the equations of the system can be used to predict the total needle shape.

\section{CAMERA And Ultrasound Image COREGISTRATION}

In order validate the model, both polynomials (1) and (2) will be referred to the same frame. Two transformation matrices are needed for the camera image and ultrasound image conversion. For the transformation matrix from the ultrasound image's pixel domain to the real-world frame, we assume that a simple affine transformation matrix, ${ }_{U}^{R} T$, will suffice as the linear transducer on the ultrasound machine results in a rectangular image. Likewise for the camera, since it is located above the center of the setup and is adjusted so that its focal plane coincides with that of the experiment we assume another affine transform matrix, denoted by ${ }_{C}^{R} T$, will suffice.

\section{A. Ultrasound Image Transformation Matrix}

The matrix ${ }_{U}^{R} T$ that transforms points from the ultrasound frame to the real-world frame is of the form

$$
{ }_{U}^{R} T=\left[\begin{array}{ccc}
{ }_{U}^{R} S_{x} \cos \phi & \sin \phi & \stackrel{R}{U} t_{x} \\
-\sin \phi & { }_{U}^{R} S_{y} \cos \phi & { }_{U}^{R} t_{y} \\
0 & 0 & 1
\end{array}\right]
$$

where ${ }_{U}^{R} t_{x}$ and ${ }_{U}^{R} t_{y}$ are the $x$ and $y$ offset, in meters, of the leftmost point of the transducer element from the base of the needle, $\phi$ is defined as the angle of the ultrasound probe relative to the closest edge of the tissue, and ${ }_{U}^{R} S_{x}$ is the pixel domain width scaling factor and ${ }_{U}^{R} S_{y}$ is the height scaling factor, see Fig. 3. The scaling elements of the matrix are constant in each tissue and can be found by measuring object distances within the tissue. The translation elements can be found through measuring the offset of the ultrasound probe. In contrast we will discuss how we calculate the value of $\phi$ in Section IV-C as this requires having the matrix ${ }_{C}^{R} T$.

\section{B. Camera Image Transformation Matrix}

The matrix ${ }_{C}^{R} T$ that transforms points from the camera frame to the real-world frame is given by

$$
{ }_{C}^{R} T=\left[\begin{array}{ccc}
{ }_{C}^{R} S_{0} & 0 & { }_{C}^{R} t_{x} \\
0 & { }_{C}^{R} S_{0} & { }_{C}^{R} t_{y} \\
0 & 0 & 1
\end{array}\right]
$$

where ${ }_{C}^{R} t_{x}$ and ${ }_{C}^{R} t_{y}$ are the $x$ and $y$ offset, in meters, of the upper leftmost point of the camera image to the base of the needle and ${ }_{C}^{R} S_{0}$ is the pixel domain scaling factor for both the $x$ and $y$ axes as the experiment is centered in the camera's field of view. As with the ultrasound matrices, the camera 


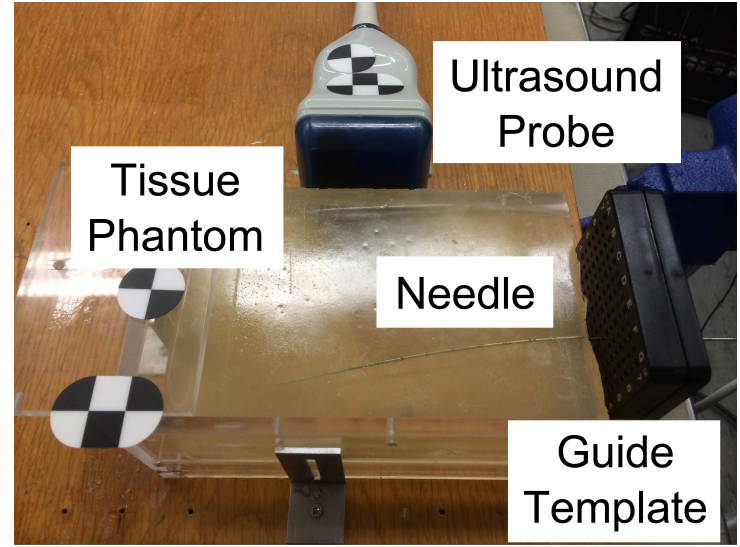

Fig. 7. Brachytherapy procedure simulation setup including ultrasound probe, phantom tissue, needle template, needle, and markers.

transformation matrices scaling and translation elements can be found through measuring known points in the physical and camera coordinate frames.

\section{Solving for the Ultrasound Image Rotation}

In order to use ${ }_{U}^{R} T$ the value of $\phi$ is required. For this experiment we will be solving for the angle $\phi$ using an iterative technique. This angle is found by comparing the results of applying the ultrasound transformation matrix ${ }_{U}^{R} T$ to the polynomial ${ }^{U} P$ (found in Section II-C), using an initial value of $\phi$, to the results of the transformation matrix ${ }_{C}^{R} T$ applied to the polynomial ${ }^{C} P$ (also found in Section II-C). The value of $\phi$ is iterated in steps of $0.1^{\circ}$ and the value that results in the best fit between both curves is chosen. The two matrices allow for the portion of the needle seen in the ultrasound image to be converted to the real-world coordinate frame and used as an input for the needle-tissue interaction model. The average registration error was less than $0.05 \mathrm{~mm}$ between the points measured in the camera frame and the points in the ultrasound frame.

\section{EXPERIMENTAL RESUlTS}

The setup for the experiment to simulate a prostate brachytherapy procedure is shown in Fig. 7. An 18-gauge by $200 \mathrm{~mm}$ prostate seeding needle (Eckert \& Zielger BEBIG $\mathrm{GmbH}$, Berlin, Germany) is inserted through a brachytherapy template (Model D0240018BK, C. R. Bard, Inc., Covington, USA) into a tissue phantom. The tissue phantom is a plastisol gel from M-F Manufacturing Co, Fort Worth, USA. The physical characteristics of the plastisol gel mimic human tissue with the added benefit that the phantom tissue transmits both visible light and ultrasound waves, allowing simultaneous capturing of images in both modalities. A portion of the needle is imaged by a 4DL14-5/38 Linear 4D transducer (different from the transrectal ultrasound probe shown in Fig. 1) which is connected to an SonixTouch ultrasound machine (Ultrasonix, Richmond, Canada). For this experiment, we only use the 2D functionality of the ultrasound probe. The entire needle is imaged from above by a XCD-SX90CR video camera (Sony Corporation of America, New York,

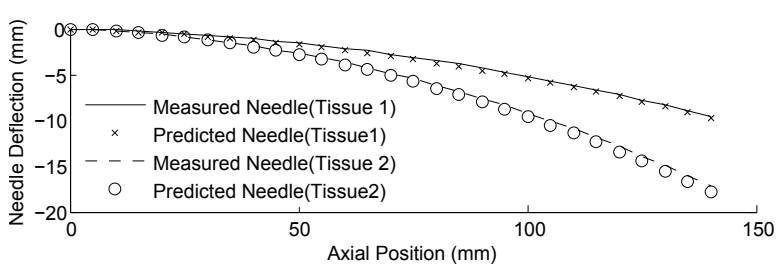

(a) Needle Shape Prediction

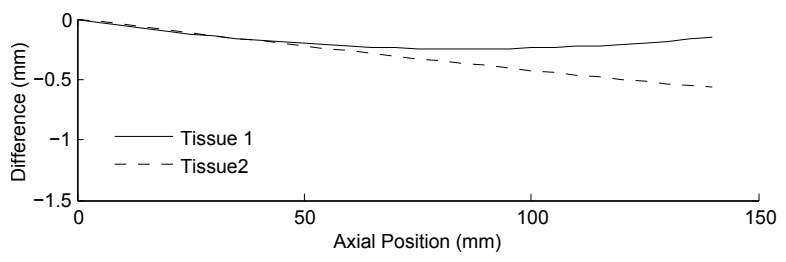

(b) Needle Shape Prediction Error

Fig. 8. Needle Shape Prediction with Error

USA). The prostate seeding needle is inserted such that it deflects in the plane imaged simultaneously by the camera and the ultrasound probe. Due to the process by which ultrasound and camera images are captured, the ultrasound probe does not interfere with the camera's field of view even while they both image the same plane. Two different tissue phantoms are used, so called Tissue 1 and Tissue 2, where Tissue 2 is considerably stiffer than Tissue 1 . Tissue 1 was created using a four to one ratio of liquid plastic to softener while Tissue 2 was created using a five to one ratio. With the needle inserted into the tissue phantom, at an insertion depth of $140 \mathrm{~mm}, 5$ images from the camera and ultrasound are taken simultaneously. Between images. the orientation of the ultrasound probe $\phi$ is changed. The images are input into the aforementioned image processing algorithms (Section II) in order to calculate the needle shape polynomials. The ultrasound polynomial equation that contains the partial observation of the needle is subsequently inserted into the needle-tissue interaction model in order to predict the entire needle shape. The predicted needle shape is compared with the measured needle shape as found by the camera in Fig. 8. Table I summarizes the needle shape prediction results for each of the 5 trails in both tissue phantoms by comparing needle tip deflection. Of note in Table I is that neither the offset location of the ultrasound probe nor the probe orientation affects the needle shape prediction results. When one compares the average measured deflection to the average deflection error both tissues have a prediction error of $20 \%$, validating that the model is tissue independent.

\section{CONCLUSION}

In this paper we have shown the feasibility of predicting the shape of an entire needle when only a portion of it has been imaged via ultrasound. Our method is based on combining image processing, coregistration of different image sources, and a needle-tissue interaction model. We have developed a new method for ultrasound image segmentation that combines thresholding, using Otsu's algorithm, 
TABLE I

Phantom Tissue Results

\begin{tabular}{cccccc}
$\begin{array}{c}\text { Experiment } \\
\text { Number }\end{array}$ & $\begin{array}{c}\text { Probe Distance } \\
\text { from Guide }\end{array}$ & $\begin{array}{c}\text { Probe } \\
\text { Orientation }\end{array}$ & $\begin{array}{c}\text { Measured } \\
\text { Deflection }\end{array}$ & $\begin{array}{c}\text { Model-Prediction } \\
\text { Deflection }\end{array}$ & $\begin{array}{c}\text { Absolute Deflection } \\
\text { Error }\end{array}$ \\
\hline & & \multicolumn{5}{c}{ Tissue 1} \\
\hline 1 & $40 \mathrm{~mm}$ & $2.1^{\circ}$ & $9.6 \mathrm{~mm}$ & $9.7 \mathrm{~mm}$ & $0.1 \mathrm{~mm}$ \\
2 & $17 \mathrm{~mm}$ & $1.4^{\circ}$ & $10.1 \mathrm{~mm}$ & $11.4 \mathrm{~mm}$ & $1.3 \mathrm{~mm}$ \\
3 & $28 \mathrm{~mm}$ & $4.1^{\circ}$ & $10.0 \mathrm{~mm}$ & $12.2 \mathrm{~mm}$ & $2.2 \mathrm{~mm}$ \\
4 & $66 \mathrm{~mm}$ & $1.5^{\circ}$ & $9.0 \mathrm{~mm}$ & $13.4 \mathrm{~mm}$ & $4.4 \mathrm{~mm}$ \\
5 & $66 \mathrm{~mm}$ & $1.7^{\circ}$ & $8.7 \mathrm{~mm}$ & $10.2 \mathrm{~mm}$ & $1.5 \mathrm{~mm}$ \\
\hline Avg & & \multicolumn{5}{c}{$9.5 \mathrm{~mm}$} & $11.4 \mathrm{~mm}$ & $1.9 \mathrm{~mm}$ \\
\hline & & $1.8^{\circ}$ & $17.6 \mathrm{~mm}$ & $12.5 \mathrm{~mm}$ & $5.1 \mathrm{~mm}$ \\
\hline 1 & $14 \mathrm{~mm}$ & $4.5^{\circ}$ & $17.3 \mathrm{~mm}$ & $18.9 \mathrm{~mm}$ & $1.6 \mathrm{~mm}$ \\
2 & $19 \mathrm{~mm}$ & $0.3^{\circ}$ & $17.5 \mathrm{~mm}$ & $23.0 \mathrm{~mm}$ & $5.6 \mathrm{~mm}$ \\
3 & $36 \mathrm{~mm}$ & $-0.8^{\circ}$ & $17.3 \mathrm{~mm}$ & $17.8 \mathrm{~mm}$ & $0.5 \mathrm{~mm}$ \\
4 & $15 \mathrm{~mm}$ & $11.9^{\circ}$ & $17.2 \mathrm{~mm}$ & $21.0 \mathrm{~mm}$ & $3.8 \mathrm{~mm}$ \\
5 & $2 \mathrm{~mm}$ & & $17.4 \mathrm{~mm}$ & $18.5 \mathrm{~mm}$ & $3.32 \mathrm{~mm}$
\end{tabular}

and RANSAC. This combination allows for the ultrasound imaged needle segment to be succinctly described as a polynomial. In addition we have extended this technique to be able to segment a needle inserted into semi-transparent tissue imaged by a camera.

As a general tool to help validate a needle-tissue interaction model, we have devised a method for coregistering ultrasound and camera images using affine transformations. To the best of the authors' knowledge, such a technique has not been presented in the literature. Potential applications of our method to medical robotics include needle pose estimation and simultaneous camera and ultrasound visual servoing.

The needle-tissue interaction model outlined here is used to predict the entire shape of the needle based on partial observation. This model only requires simple mechanical characteristics of the needle and is independent of tissue characteristics and needle-tissue force interactions. Furthermore, the input to this model can be provided by either an ultrasound or camera image. The algorithm as outlined by this paper has been validated in phantom tissue. The experimental results have shown that the needle shape can be estimated with an accuracy greater than $5.6 \mathrm{~mm}$. In our future work, we intend to integrate this routine into a brachytherapy needle insertion assistant robot to allow for real-time needle deflection estimation and correction.

\section{REFERENCES}

[1] J. E. Dawson, T. Wu, T. Roy, J. Y. Gu, and H. Kim, "Dose effects of seeds placement deviations from pre-planned positions in ultrasound guided prostate implants." Radiotherapy And Oncology: Journal Of The European Society For Therapeutic Radiology And Oncology, vol. 32, no. 3, pp. $268-270,1994$.

[2] S. Misra, K. Reed, B. Schafer, K. Ramesh, and A. Okamura, "Mechanics of flexible needles robotically steered through soft tissue." International Journal of Robotics Research, vol. 29, no. 13, pp. 1640 - 1660, 2010.
[3] D. De Lorenzo, Y. Koseki, E. De Momi, K. Chinzei, and A. M. Okamura, "Coaxial needle insertion assistant with enhanced force feedback." IEEE Transactions on Biomedical Engineering, vol. 60, no. 2, pp. 379 - 389, 2013.

[4] S. P. Dimaio and S. E. Salcudean, "Needle steering and motion planning in soft tissues." IEEE Transactions on Biomedical Engineering, vol. 52, no. 6, pp. $965-974,2005$.

[5] M. A. Fischler and R. C. Bolles, "Random sample consensus: A paradigm for model fitting with applications to image analysis and automated cartography," Communications of the ACM, vol. 24, no. 6 , pp. 381-395, Jun. 1981.

[6] M. Kaya and O. Bebek, "Needle localization using gabor filtering in 2D ultrasound images," in In Proceedings of the IEEE International Conference on Robotics and Automation, 2014, pp. pp. 4881-4886.

[7] M. Ding and A. Fenster, "A real-time biopsy needle segmentation technique using Hough transform.” Medical Physics, vol. 30, no. 8, pp. $2222-2233,2003$.

[8] S. Okazawa, R. Ebrahimi, J. Chuang, R. Rohling, and S. Salcudean, "Methods for segmenting curved needles in ultrasound images." Medical Image Analysis, vol. 10, no. 3, pp. 330 - 342, 2006.

[9] P. Novotny, J. Stoll, N. Vasilyev, P. del Nido, P. Dupont, T. Zickler, and R. Howe, "Gpu based real-time instrument tracking with threedimensional ultrasound." Medical Image Analysis, vol. 11, no. 5, pp. 458 - 464, 2007.

[10] M. Ding and A. Fenster, "Projection-based needle segmentation in 3D ultrasound images." Computer Aided Surgery, vol. 9, no. 5, pp. 193 201, 2004.

[11] M. Uherčík, J. Kybic, H. Liebgott, and C. Cachard, "Model fitting using RANSAC for surgical tool localization in 3-D ultrasound images." IEEE Transactions on Biomedical Engineering, vol. 57, no. 8, pp. 1907 - 1916, 2010.

[12] N. Otsu, "A threshold selection method from gray-level histograms," Systems, Man and Cybernetics, IEEE Transactions on, vol. 9, no. 1, pp. 62-66, Jan 1979.

[13] R. C. Gonzalez and R. E. Woods, Digital image processing. Upper Saddle River, N.J. : Prentice Hall, 2002.

[14] O. Goksel, E. Dehghan, and S. E. Salcudean, "Modeling and simulation of flexible needles," Medical engineering \& physics, vol. 31, no. 9, pp. 1069-1078, 2009. 